\title{
Absolute versus relative presentation of tcdB Clostridium difficile biomarker
}

\author{
Ivan Brukner ${ }^{1,2,3}$, Shaun Eintracht ${ }^{3,4}$, Vincenzo Forgetta ${ }^{2}$, Alan Spatz ${ }^{2,3,5}$, Andreas I Papadakis ${ }^{3}$ and Matthew Oughton $^{1,3,4 *}$ \\ ${ }^{1}$ Department of Medical Microbiology, Jewish General Hospital, Quebec, Canada \\ ${ }^{2}$ Lady Davis Institute for Medical Research, Quebec, Canada \\ ${ }^{3}$ McGill University, Faculty of Medicine, Montreal, Quebec, Canada \\ ${ }^{4}$ Department of Medicine, Jewish General Hospital, Quebec, Canada \\ ${ }^{5} \mathrm{McGill}$ University, Department of Pathology, Quebec, Canada
}

\begin{abstract}
Clostridium difficile infection is the major etiologic agent of antibiotic-associated pseudomembranous colitis, a disease that can be fatal if unrecognized, or untreated. On average, there are 15000 deaths and 500000 new cases per year, in the USA. Diagnostic biomarkers currently used are the tcdB gene, or its gene product (toxin B). Clinical interpretation of the assay is particularly challenging: (1) biomarker detection is possible without manifestation of symptoms and (2) missing biomarker due to assay sensitivity limitations can be fatal. To resolve clinical uncertainty, quantitative analysis has been considered. Despite multiple efforts, a quantitative tcdB/toxB threshold with a meaningful clinical impact has yet to be established. Herein we shed light as to why mass/volume-based normalisations were fruitless in the past. Specifically, measuring total bacterial flora (using "universal bacterial" $16 \mathrm{~S}$ qPCR rDNA assay) to calculate relative abundance of $C$ difficile, we demonstrate a strong and significant negative correlation between tcdB biomarker of $C$ difficile $\left(\mathrm{R}^{2}=0.73, \mathrm{~N}=227, \mathrm{P}=10^{-39}\right)$ and the rest of gut microorganisms. The new parameter $(\mathrm{Cq}$ (toxB)-Cq $(16 \mathrm{SrDNA}))$ is calculated from two biomarkers, is independent of sampling variability and inherently incorporates the destructive character of $C$ difficile on the rest of micro-flora. By incorporating relative abundance of tcdB (in context of the total bacterial flora), and correcting for "biomass wash-out/dilution effects of acute diarrhea , these biomarkers could collectively enhance the predictive value of CDI testing.
\end{abstract}

\section{Introduction}

Bacterial biomass is the major component (25-54\% of dry solids) of the organic fraction of the feces [1]. Stool, stool swabs, or rectal swabs are types of samples used in molecular diagnostics of diarrhea. Sample processing involves various technical variabilities. For instance, sampling of heterogeneous gut microbiome content of the stool can be improved by homogenizing the stool prior to subsampling and by using standard volume input (use of 10 microliter microbiology loop) prior to sample processing [2]. However, despite all pre-cautions, intestinal bowel material is "differentially diluted" among different patients, due to case dependent differential "biomass wash-outs", during presentation of acute diarrheal symptoms, the total bacterial load could vary up to few $\log$ values [3]. Whenever pre-analytical sampling variability is large $[1,2,4-8]$ and quantification is intended to establish the clinical status of the patient, normalization based on sample mass, or sample volume is not effective.

Recent reports demonstrate that cycle threshold values $(\mathrm{Cq})$ of tcdB assay overlap between symptomatic and asymptomatic patients. Median Cq value of the tcdB PCR in toxigenic C. difficile-positive healthy individuals is significantly higher than for symptomatic patients, indicating that lower tcdB gene load is present in the stool of persons with asymptomatic colonization. However, a numerical consensus is lacking, and future diagnostic strategy is not conclusive [9-16]. Despite the evidence that both qPCR and novel "ultrasensitive" enzyme immunoassay technologies possess single molecule resolution for CDI detection, the pre-analytical variability of sampling, may at least in part explain the current inability to establish sharp quantitative thresholds with meaningful clinical value [12,17-20]. Since normalization of diarrhea based on mass or volume, does not correct for the past wash-outs of intestinal content during acute CDI phase, symptomatic and asymptomatic toxB/tcdB positive samples will provide partially overlapping absolute quantitative values. We applied relative normalization of Cq values, a strategy well described and promoted by "typical" gene expression studies and summarized in MIQE guidelines $[21,22]$. Herein, we report that "the rest of bacterial microorganisms" shows negative correlation with tcdB biomarker, thus presenting valuable biomarker capable of clearly identifying samples with extremely high $\mathrm{tcdB}$ burden, independent from the absolute concentration of tcdB per volume and/or mass of fecal material.

\section{Material and methods}

Sample processing was equivalent to the one described in BD GeneOhm TM C diff Assay Manual (https://www. bd.com/resource). All oligonucleotides were synthesized by Integrated DNA Technologies (IDT) (Iowa, US): tcdB primers MTO2F (5'TGCAGCCAAAGTTGTTGAAT3') and MTO2R (5'GCTCTTTGATTGCTGCACCT3'), and probe MTO2P (/56FAM/TCTGAAGGA/ZEN/TTACCTRTAATT GCAA/3IABkFQ/; QuantiNova Probe PCR kit (Cat No./ID: 208254, Qiagen, Canada);

${ }^{*}$ Correspondence to: Ivan Brukner, Jewish General Hospital, 3775 Cotes des Neiges, Montréal, Room E-613, Québec, H3T 1E2, Canada, Tel: +514 3408222 E-mail: ibrukner@jgh.mcgill.ca

Received: March 14, 2019; Accepted: March 26, 2019; Published: April 02, 2019 
The full sequence of Inhibition Control Target was (5'TGCAGCCAA AGTTGTTGAATGCAATGGTCCCAATGGCTAACGCGCAGAG CCTTCAGGTCAGAAATTTTTGCCATC CGAGACATCAGGTG CAGCAATCAAAGAGC3'), detected by hydrolysis probe /56-FAM/ TTCTGACCT/ZEN/GAAGGCTCTGCGCG/3IABkFQ/. Described tcdB $\mathrm{qPCR}$ assay was validated with multiple commercial assays in the past (C difficile Quick Check Complete, Alere; BD Max, BD and $\mathrm{BD}$ GeneOhm, BD) and used over last 10 years in our institution to detect symptomatic patients which are positive for tcdB biomarker. The relative normalization of samples was obtained using universal $16 \mathrm{~S} \mathrm{rDNA}$ amplicons, as a measure of total bacterial load, as described [23]. The qPCR was performed on Roche Light Cycler 480 instrument, following PCR program: hold at $94^{\circ} \mathrm{C}$ for 2 minutes and cycling of $45 \mathrm{x}$ $\left(94^{\circ} \mathrm{C}, 10 \mathrm{sec}\right.$, and following by priming, elongation and acquisition of fluorescence at $50^{\circ} \mathrm{C}$ for 20 seconds). The RFU (Relative Fluorescence Units) versus cycles curves were visually analyzed. Internal PCR positive control was genomic DNA from C. difficile ATCC 43255, adjusted to produce positive and stable Cq values. LC480 software (V 1.5.1.62 SP3) was used to calculate $\mathrm{Cq}$ values using second derivate analyses and high sensitivity mode. Decisions of the result was made by operator (positive/negative/ inhibition), based on interplay between results of tcdB and/or inhibition assays and curve shape, and/or generated $\mathrm{Cq}$ value. Total bacterial load quantifications were performed on tenfolddiluted sample (comparing to equivalent tcdB assay) to assure that inhibition of $16 \mathrm{~S}$ rDNA reaction was not a dominant factor in defining $\mathrm{Cq}$ value. The difference in $\mathrm{Cq}$ values among $16 \mathrm{~S}$ and tcdB reactions (each done in separate wells) was calculated, after correction for sample dilution.

\section{Results and discussion}

The 227 clinical stool samples, declared as positive for tcdB by laboratory developed test (tcdB qPCR), were collected in 2015 in the Clinical Microbiology Laboratory, Jewish General Hospital. Total bacterial load ( $\mathrm{Cq}$ qPCR values of $16 \mathrm{~s} \mathrm{rDNA}$ ) was measured in these samples as second biomarker. The average value for $\mathrm{Cq}$ of $\mathrm{tcdB}$ in this group of patients was 28.8 , covering a minimum-maximum range of 20.5 to 38.6. The standard deviation among repetitive measurements for any of these assays was lower than $2 \mathrm{Cq}$ units. The second biomarker was chosen with the intention of normalizing pre-analytical sample variability, i.e. to calculate relative abundance of $C$. difficile in total stool flora. The $\mathrm{Cq}$ values of total bacterial load among collected clinical samples (16S rDNA qPCR) showed a distribution with an average of $\mathrm{Cq}=16.6$ and minimum-maximum range of 12.2 to 23.7. Using paired numerical data (tcdB and $16 \mathrm{~S}$ rDNA) for each sample, a relative abundance of $C$. difficile was calculated, thereby correcting for sampling variations which are beyond control (e.g. stool mass, stool volume and frequency of defecation). When absolute Cq values of tcdB and relative values were plotted against each other, a strong correlation $\left(\mathrm{R}^{2}=0.73, \mathrm{~N}=227, \mathrm{P}=10^{-39}\right)$ was obtained. The number $\mathrm{Cq}(\mathrm{tcdB})$ minus $\mathrm{Cq}$ (16S rDNA) reflects the relative abundance of tcdB in total flora. The average value for this number was 12.9 , with a minimum-maximum range between 2.2 to 20.2. The bigger this number is, the smaller the relative abundance of $C$ difficile is. If this number was kept constant and independent from the concentration of tcdB biomarker, we would assume that $C$. difficile is in commensal relation with the rest of bacterial microorganisms. However, data suggests that overgrowth of $C$. difficile strongly suppresses the rest of gut flora (by competition), suggesting aggressive overgrowth of gut eco-system by the pathogenic species. The relative abundance of toxicogenic $C$. difficile in asymptomatic cases usually represents a minor fraction [24]. For example, a recent high-resolution profiling of the gut microbiome reveals the extent of C. difficile burden, suggesting that abundance of $C$. difficile among CDI patients is $1.78 \%$ or higher, which is quite different from healthy controls $(0.008 \%)$ [25]. In summary, by measuring relative abundance of tcdB against total bacterial flora, the method corrected for a "biomass wash-out/dilution" effects of the fecal samples during acute diarrhea and captured the individual's ratio of $C$. difficile vs. the rest of the microbiota. The combination of these two biomarkers could enhance the predictive value of test for CDI detection.

\section{Acknowledgments}

The thanks go to Mm Coleen Delisle and Dr Jack Travors, for critical opinions and suggestions, Dr Yves Longtin for continual interest in the subject of technological advances and Dr Andre Dascal for his efforts in establishing bridges between clinical microbiology and novel molecular technologies. Work was partially funded by CIHR (application number 345267). We want to thank Nadia Nour, PhD for commercialization efforts.

\section{References}

1. Rose C (2015) The characterization of feces and urine: A review of the literature to inform advanced treatment technology. Crit Rev Environ Sci Technol 45: 1827-1879. [Crossref]

2. Gorzelak MA (2015) Methods for improving human gut microbiome data by reducing variability through sample processing and storage of stool. PLoS One 10: e0134802. [Crossref]

3. Brukner I (2015) Assay for estimating total bacterial load: relative qPCR normalisation of bacterial load with associated clinical implications. Diagn Microbiol Infect Dis 83: 1-6. [Crossref]

4. Begum YA (2018) In Situ analyses directly in diarrheal stool reveal large variations in bacterial load and active toxin expression of enterotoxigenic escherichiacoli and vibrio cholerae. mSphere 3: 1-10. [Crossref]

5. Bliss DZ (1999) Comparison of subjective classification of stool consistency and stool water content. J Wound Ostomy Continence Nurs 26: 137-141. [Crossref]

6. Lordani CR, Eckert RG, Tozetto AG, Lordani TV, Duarte PA (2014) The knowledge of intensive care professionals about diarrhea. Rev Bras Ter Intensiva 26: 299-304. [Crossref]

7. Guenter PA, Sweed MR (1998) A valid and reliable tool to quantify stool output in tubefed patients. JPEN J Parenter Enteral Nutr 22: 147-151. [Crossref]

8. William HB, Kathleen R, Michael O'Connor H (2017) Mild, Moderate, or Severe Diarrhea. https://www.healthlinkbc.ca/health-topics/sig18272. Accessed on 2017.

9. Anikst VE (2016) Organism burden, toxin concentration, and lactoferrin concentration do not distinguish between clinically significant and nonsignificant diarrhea in patients with Clostridium difficile. Diagn Microbiol Infect Dis 84: 343-346.

10. Fang FC, Polage CR, Wilcox MH (2017) Point-Counterpoint: what is the optimal approach for detection of clostridium difficile infection? J Clin Microbiol 55: 670-680. [Crossref]

11. Kamboj M (2018) Potential of real-time PCR threshold cycle (CT) to predict presence of free toxin and clinically relevant $\mathrm{C}$. difficile infection (CDI) in patients with cancer. J Infect 76: 369-375.

12. Kim HN (2018) Toxin positivity and tcdB gene load in broad-spectrum Clostridium difficile infection. Infection 46: 113-117.

13. Madden GR, Poulter MD, Sifri CD (2018) PCR cycle threshold to assess a diagnostic stewardship intervention for C. difficile testing. J Infect 78: 158-169. [Crossref]

14. Senchyna F, Gaur RL, Gombar S, Truong CY, Schroeder LF, et al. (2017) Clostridium difficile PCR Cycle Threshold Predicts Free Toxin. J Clin Microbiol 55: 2651-2660. [Crossref]

15. Wilmore S, Goldenberg SD (2018) Potential of real-time PCR threshold cycle (CT) to predict presence of free toxin and clinically relevant $\mathrm{C}$. difficile infection (CDI) in patients with cancer: A reply. J Infect 76: 424-426. 
16. Origuen J (2019) Toxin B PCR amplification cycle threshold adds little to clinical variables for predicting outcomes in clostridium difficile infection: A retrospective cohort study. J Clin Microbiol 57: 1-10. [Crossref]

17. Lashner BA (1986) Clostridium difficile culture-positive toxin-negative diarrhea. Am J Gastroenterol 81: 940-943.

18. Polage CR, Turkiewicz JV, Cohen SH (2016) The never-ending struggle with laboratory testing for Clostridium difficile infection. J Comp Eff Res 5: 113-116.

19. Pollock NR (2016) Ultrasensitive detection and quantification of toxins for optimized diagnosis of clostridium difficile infection. J Clin Microbiol 54: 259264. [Crossref]

20. Sandlund J (2018) Ultrasensitive detection of clostridioides difficile toxins a and b by use of automated single-molecule counting technology. J Clin Microbiol 56: 1-10. [Crossref]
21. Bustin S (2017) The continuing problem of poor transparency of reporting and use of inappropriate methods for RT-qPCR. Biomol Detect Quantif 12: 7-9. [Crossref]

22. Bustin S, Nolan T (2017) Talking the talk, but not walking the walk: RT-qPCR as a paradigm for the lack of reproducibility in molecular research. Eur J Clin Invest 47 : 756-774. [Crossref]

23. Glisovic S (2018) Rectal swab screening assays of public health importance in molecular diagnostics: Sample adequacy control. J Infect Public Health 11: 234237.

24. Vincent C (2016) Bloom and bust intestinal microbiota dynamics in response to hospital exposures and Clostridium difficile colonization or infection. Microbiome 4: 1-12.

25. Daquigan N (2017) High-resolution profiling of the gut microbiome reveals the extent of Clostridium difficile burden. NPJ Biofilms Microbiomes 3: 25-35.

Copyright: (2019 Brukner I. This is an open-access article distributed under the terms of the Creative Commons Attribution License, which permits unrestricted use, distribution, and reproduction in any medium, provided the original author and source are credited. 